\title{
II JORNADAS DE FILOSOFÍA Y DESARROLLO PERSONAL. Filosofía PRÁCTICA Y MUSICOTERAPIA
}

\author{
ÓLIVER ÁLVAREZ VALLE \\ Universidad de Valladolid \\ Grupo Penicilina Filosófica de Valladolid. \\ penicilinafilosofica@yahoo.es
}

RECIBIDO: 12 DE MARZO DE 2010

ACEPTADO: 2 DE MAYO DE 2010

\section{Introducción}

Desde el 6 al 9 de noviembre de 2009, algunos de los más importantes promotores de la Filosofía Práctica, de España y del mundo, se reunieron en el Ateneo, en el centro de Madrid. Mostraron una introducción a este creciente movimiento filosófico, con un día de conferencias abiertas y tres días dedicados a talleres, incluyendo entre los enfoques estudiados, la base del uso filosófico de la musicoterapia.

Aunque la iniciativa de celebrar estas jornadas partió de la sección de Filosofía del Ateneo de Madrid, todo el peso de la organización fue llevado por Victoria Caro, licenciada en Filosofía y miembro titulado de ASEPRAF (Asociación Española para la Práctica y el Asesoramiento Filosóficos). Gracias a su empeño, y a la aportación de todos los conferenciantes, se pudo disfrutar de un acercamiento global a esta corriente filosófica.

\section{Viernes: El arte de preguntar, taller impartido por Óscar Brenifier ${ }^{1}$.}

Los talleres de Óscar Brenifier son una puesta en práctica de su "arte de preguntar", desde el primer minuto, los asistentes se someten a la dinámica de responder de forma concisa y sin ambigüedades. Ésa es la

\footnotetext{
${ }^{1}$ Fundador del Institut de Practiques Philosophiques.
} 
base de su práctica filosófica, aplicable en todas sus vertientes, no sólo en los talleres, sino también en los cafés filosóficos, en el aula y en la consulta individual o de pareja. En lugar de exponer primero una base teórica, el aprendizaje se ha de producir sobre la marcha del diálogo. Todo ello es coherente con la metodología pedagógica que ha propuesto en libros como "El diálogo en clase"2. En nuestro caso el interés estaba en la mecánica y los recursos técnicos del cuestionamiento, y en sus fundamentos teóricos, ocasionalmente detallados cuando afloraban en la práctica.

Comenzamos con un EJERCICIO DE INTERPRETACIÓN, consistente en contestar por escrito a la pregunta “¿por qué están aquí?” y analizar en común las respuestas. Fue una forma de poner en marcha el diálogo, pero el objetivo no estaba en terminar el ejercicio (los incisos fueron todo lo largos que era necesario) sino centrarnos en las palabras "fáticas", las interjecciones, los lapsus, los errores, etc. esas "pequeñas cosas que son reflejo de lo causal", aplicando el principio de razón suficiente de Leibniz. En palabras del propio Brenifier durante el taller: "En una sola palabra puede estar tu visión del mundo", o "lo que importa no es la palabra, es el ser". A partir de esos detalles, normalmente no percibidos en el discurso, se inicia con decisión una senda, "como Descartes a través del bosque", que puede llevarnos a algún sitio o no, pero, "como en la apuesta de Pascal", tenemos poco que perder. El enfoque en los errores, sumado a la regla de anunciar antes de cada intervención de qué tipo va a ser ésta (respuesta, pregunta, comentario...) provoca una mayor concentración en el discurso, propio y ajeno, y una barrera que frena la charla no reflexionada. "Hay que parar de hablar para luego poder hablar", porque "la vida humana es la producción de una imagen ante la que haces cualquier sacrificio", y la charla cotidiana termina siendo una "conversación de hologramas", por ello "la Filosofía es un trabajo iconoclasta". Pero nos identificamos con esa imagen ilusoria $\mathrm{y}$, por eso, "el ojo que da más miedo no es el del otro, es el propio" y "es un regalo envenenado obligarte a verte a ti mismo". Ilustró este punto con la metáfora "del mono malayo", que mete la mano en una jaula para coger un coco y, aunque no puede sacarla sin soltarlo, no lo suelta aunque

${ }^{2}$ BRENIFIER, Óscar: El diálogo en clase, Ediciones Idea, S. C. Tenerife, 2005 
vea venir los cazadores a por él. También a los humanos nos cuesta "soltar" y dejar de defender nuestra imagen. Pero si lo conseguimos, nos liberamos intelectualmente de esa trampa:

"El arte de preguntar libera el pensamiento de estar obligado a tener una meta".

La práctica de Brenifier deja en suspenso la teleología, "el sabio no tiene deseo". En ese sentido improvisó el "neologismo" "contra-doxá", ya que es algo que parece ir contra el "sentido común". Algunos recursos técnicos que utilizó y explicó, para hacer conscientes esas nuevas posibilidades de pensamiento fueron:

- El planteamiento de antinomias como ejercicio ("nietzscheano") de transvaloración. Buscar aspectos positivos y negativos en ambos opuestos obliga a dejar de juzgar, y clasificar uno como bueno y otro como malo. Un útil recurso, pedagógico ${ }^{4}$ y para la consulta.

- Transposiciones o ejemplos imaginarios, para distanciar y relajar la resistencia.

- El paso de concreto a general, que también elude la resistencia, y viceversa, para volver a implicar.

- Profundizar y problematizar, respectivamente para planteamientos que se pierden en demasiadas posibilidades $\mathrm{y}$ para los excesivamente simplificadores. Simplificar y generalizar son dos mecanismos de defensa opuestos, por ello afirmó: "mi trabajo es hacer simples los pensamientos complicados $\mathrm{y}$ complicados los simples".

En la sesión de tarde realizamos dos ejercicios más:

EJERCICIO PARA PREGUNTAR, "FILÓSOFO COLECTIVO”. Un supuesto consultante responde las preguntas que elige el grupo. Sobre la marcha vimos, entre otros muchos, recursos como:

- No añadir en las preguntas supuestos o prejuicios propios. La "alienación", en el sentido de "ser nadie", es necesaria en el

\footnotetext{
${ }^{3}$ Interesante el desarrollo de este epígrafe en el artículo expuesto en su web, BRENIFIER, Óscar: "Filosofar es dejar de vivir", disponible on-line en http://www.brenifier.com/espanol/filosofar_es_dejar_de_vivir.htm (último acceso 28 de noviembre de 2009).

${ }^{4}$ BRENIFIER, Óscar: Ni sí ni no. Un libro para entender los grandes contrarios del pensamiento, Ediciones SM, Madrid, 2008.
} 
filósofo asesor. "Filosofar es inhumano, exige no tener ninguna visión".

- En una contradicción aislar y definir los términos por separado.

- Asegurarse de que el consultante sigue el hilo mediante preguntas sin grandes saltos.

- Avanzar tornando en preguntas las afirmaciones más tajantes, con preguntas "diabólicas" del tipo "¿cómo lo sabes?".

Brenifier propuso que, como variante de este ejercicio, preguntar y responderse a sí mismo por escrito, puede ser una buena práctica de asesoramiento, con uno mismo como asesorado, ya que la escritura implica un compromiso con la respuesta, y posibilita su análisis.

EJERCICIO DE EVALUACIÓN por escrito con tres comentarios y tres preguntas por cada asistente. En esta última fase del taller pudimos escuchar nociones sobre las que reflexionar como: "Filosofar es descubrir nuestra incoherencia", "el trabajo del filósofo es decepcionar", "el brujo no vive en el pueblo, sino en sus límites", o la platónica "verdad y amistad no van bien juntas".

Finalizó un taller, como suelen ser los de Brenifier, sumamente intenso. Una muestra de un verdadero arte, el arte de preguntar, que saca a la luz ese sorprendente modo de pensar subyacente, del que muy pocas veces tenemos la oportunidad de hacernos conscientes. Su descubrimiento no siempre es agradable y quizá la mejor forma de disfrutar de esa toma de conciencia, de ese "descubrimiento de nuestra incoherencia", sea con humor. Como suele decir el propio Brenifier en tales momentos: “¿Divertido, no?”

\section{Sábado: Conferencias abiertas al público. Sesión de mañana, Salón de Actos.}

Itziar Álvarez, como soprano, y Montse Muñoz Ávila, al piano, intercalaron entre las conferencias algunas piezas musicales, seleccionadas y comentadas brevemente por Mariano Betés de Toro. 


\section{Presentación de la jornada de conferencias, por JOSÉ LUIS ABELLÁN ${ }^{5}$.}

El profesor Abellán mostró lo oportuno y relevante de estas jornadas como muestra de un incipiente nuevo ciclo de la filosofía, tras el agotamiento del que se inició en Grecia, desde la práctica y el desarrollo personal, y que terminó en su opuesto de la crisis de la razón instrumental plegada a lo material, pasando por el momento de "éxito y perdición" que supuso la segunda mitad del siglo XIX en Alemania con la institucionalización de la filosofía entendida exclusivamente como conjunto de grandes sistemas de razón discursiva. La obra de Abellán se rebela contra esa concepción restringida, y reivindica la filosofía española y el concepto de "exilio filosófico" ". Espera que esa labor dé sus frutos porque puede que la crisis que vivimos actualmente esté originada, precisamente, por la mencionada crisis de la filosofía.

Abellán ve en estas jornadas una señal del nuevo ciclo filosófico. Si el anterior, desde Aristóteles, dio la prioridad al sentido de la vista, éste nuevo ciclo debería estar basado más en el oído, como propuso ya María Zambrano, recuperando la tradición órfico-pitagórica. "Hay que aprender a escuchar" por encima del exceso de información y ruido que nos rodea. Eso sólo es posible recuperando el "sentido del silencio" y escuchando música. Por ello el surgir de la musicoterapia, y su incorporación a estas jornadas, es un indicio de tanta relevancia. Éstas fueron las palabras, profundas y a la vez asequibles a todo el público, del profesor Abellán, que compartió con nosotros la bonita anécdota de la frase con la que María Zambrano le dedicó un libro: "A José Luis Abellán, que oye crecer la hierba".

Introducción a la Práctica Filosófica y al Asesoramiento Filosófico, por JOSÉ LUIS ROMERO'.

En esta necesaria y completa introducción, abordó los siguientes puntos:

\footnotetext{
${ }^{5}$ Catedrático de Historia de la Filosofía en la Universidad Complutense de Madrid.

${ }^{6}$ Mencionó su texto ABELLÁN, José Luis: El exilio como constante y como categoría, Biblioteca Nueva, Madrid, 2001.

${ }^{7}$ Asesor filosófico y Master Oficial en Psicoanálisis y Filosofía de la Cultura.
} 
- NOMBRE. "Práctica Filosófica" fue, entre todas las usadas actualmente, la denominación que propuso como más ajustada para esta corriente, dado que recalca la dimensión práctica que la caracteriza.

- TIPOS. Citó a Gabriel Arnaiz ${ }^{8}$ para hacer una clasificación en cinco tipos: Diálogos socráticos, planteados a principios del s.XX por Leonard Nelson; Filosofía para Niños $(\mathrm{FpN})$, corriente pedagógica iniciada por Mathew Lipman en los 70; cafés y talleres filosóficos; asesoramiento filosófico a particulares; $y$ asesoramiento a organizaciones.

- OBJETIVO. "Recuperar la aplicación a la vida, característica de la Filosofía desde sus inicios".

- BASE. Hallar incoherencias, internas y externas, de la filosofía, implícita y operativa, de cada persona.

- MÉTODO. Diálogo mayéutico para la visualización y reconfiguración por parte del consultante de su propio marco de pensamiento. Puede complementarse con lecturas filosóficas y otros recursos.

- HISTORIA. Aunque con antecedentes anteriores, Gerd Achenbach es el pionero de esta corriente, en 1981 funda la Asociación Alemana de Práctica Filosófica, la primera, que en 1998 se promociona en Sociedad Internacional. En 1995 Ran Lahav y Lou Marinoff convocan el 1er Congreso Mundial de Prácticas Filosóficas. En 1999 el best-seller de Marinoff ${ }^{9}$ la lleva al gran público. En la actualidad ya está incorporada a los estudios de postgrado en universidades de distintos países.

- ENTORNO IBEROAMERICANO. Entre 1998 y 2000 iniciaron sus actividades la asociación Búho Rojo, en Perú, Roxana Kreimer en Argentina, el Grupo Etor y el Gabinete Pharos en España. En 2001 Mónica Cavallé se convirtió en la primera presidenta de la Asociación Española para la Práctica y el Asesoramiento Filosóficos (ASEPRAF). La única revista especializada en España fue editada

\footnotetext{
8 ARNAIZ, Gabriel: "El giro práctico de la Filosofía", en Diálogo Filosófico, número 68, Madrid, 2007. Págs. 170-206.

${ }^{9}$ MARINOFF, Lou: Más Platón y menos Prozac, Ediciones B, Barcelona, 2000.
} 
por el Grupo Etor ${ }^{10}$, vinculado a la Universidad de Sevilla, que ofrece un título de Experto en la materia que, junto con el Máster en Práctica Filosófica y Gestión Social de la Universidad de Barcelona, son los únicos títulos universitarios españoles en el área. Obras pioneras en España han sido la de Luis Cencillo ${ }^{11}$, y la tesis de José Luis Barrientos $^{12}$, quien organizó el 1er Congreso Iberoamericano y $8^{\circ}$ Congreso Mundial de Prácticas Filosóficas.

- CONTROVERSIAS. ¿Qué filosofía sustenta la Práctica Filosófica, ¿qué relación hay entre Filosofía y Práctica?, ¿y con la Psicología?, son algunas de las cuestiones aún por definir en este novedoso campo.

Sabiduría y complacencia. Filosofar para protegerse de sí mismo, por Óscar Brenifier.

Brenifier buscó, como es habitual en él, la interacción con la audiencia, en tres fases:

- EN LA PRIMERA pidió participación, y el hecho de que se alzasen pocas manos, sirvió como respaldo para la tesis del título, "la gente gasta mucha energía en defenderse", y "las preguntas se detectan como una amenaza", ya desde la escuela. Puso el ejemplo de que los alumnos bajan las manos cuando les pregunta por qué las han levantado. "Siento decir que mataron a Sócrates, y fue por eso. Yo me voy, pero él se quedaba". Para hablar de la complacencia y esa defensa de uno mismo usó algunas fábulas de Nasrudín, como la del amigo poeta que le insultaba airadamente por haberle dicho que no le gustaba su poesía, "no me gusta tu poesía pero tu prosa es excelente", fue la respuesta del mulá a esos insultos. También citó a Wittgenstein y afirmó que "filosofar es luchar con la fascinación que ejercen sobre nosotros algunas formas de expresión"13, cada época tiene sus palabras sagradas, como espantapájaros y, en la nuestra,

\footnotetext{
${ }^{10}$ Precedente de la actual HASER.

${ }^{11}$ CENCILLO, Luis: Cómo Platón se vuelve terapeuta: Respuesta a Marinoff, Ediciones Fundación, Madrid, 2002.

12 BARRIENTOS RASTROJO, José: “Tesis doctoral sobre Filosofía Aplicada”, disponible on-line en http://issuu.com/jbbr/docs/tesis (último acceso 1 de diciembre de 2009).

${ }^{13}$ WITTGENSTEIN, Ludwig: Los cuadernos azul y marrón, Tecnos, Madrid, 2009.
} 
"respeto" es una de ellas. No propuso abandonar esas expresiones, sino parar su "fascinación". Se apoyó en la expresión de Abellán, en la primera de las conferencias, "oír el silencio", y la relacionó con el concepto chino del "sabor insípido" que permite apreciar los demás. "El filósofo te muestra lo divertido de tu problema", pero eso puede ser tan duro como "atravesar un muro de fuego", como el que, según Dante, separa el Purgatorio del Cielo.

- LA SEGUNDA interacción fue con Elena, una voluntaria del público. Al poco tiempo Brenifier hizo un inciso: “¿Por qué cuando respondo habla con la vecina?. Voy a decir algo terrible: Todo Elena está en ese gesto". Así mostró tanto el mensaje del taller del viernes, como su provocadora forma de trabajar: "El filósofo práctico invita a una postura impensable, para poder verse a uno mismo".

- LA TERCERA respondió a la pregunta que servía de antetítulo a la charla: “Es posible filosofar con todo el mundo: niños y adultos?”. Dos niños subieron al escenario y les pidió una pregunta. "¿Puedes hablar de tus libros para niños?", dijo Andrés, uno de ellos. Brenifier trabajó con él para que descubriese por sí mismo la diferencia entre una auténtica pregunta y una petición. El otro niño, Simón, terminó llamando "mentiroso", a propuesta de Brenifier, a uno de los niños del público, que había levantado la mano para fingir, según la hipótesis del propio Simón, que sabía responder y luego no lo hizo. Ambos niños rieron, pero a los adultos no les gusta ver eso, según la experiencia de Brenifier. De hecho, un adulto del público pidió la palabra antes de terminar para aclarar que "no necesariamente era mentiroso". Brenifier le reprochó que así no se les hace honor, al niño y su hipótesis.

Más allá de la Filosofía. Desde el análisis filosófico a la comprensión espiritual, por RAN LAHAV $V^{14}$

En pie y en inglés, con traducción consecutiva, Lahav comenzó con la cuestión de “¿en qué puede la Práctica Filosófica contribuir al mundo?”. En principio no para divertir, curar ni proporcionar satisfacción. "El

\footnotetext{
${ }^{14}$ Asesor filosófico y promotor del 1er Congreso Mundial de Prácticas Filosóficas.
} 
filósofo no cuadra dentro del juego de la oferta y la demanda porque la esencia de la Filosofía no es jugar a lo que juegan las personas, sino reflexionar sobre esos juegos desde fuera". Aquí expuso su versión de la alegoría platónica de la caverna para referirse a que "el papel del filósofo no es hacer las sillas de la caverna más cómodas, o las sombras más bonitas, sino mostrar que todo eso es una caverna, y que se puede salir". Aunque la luz de fuera haga daño, hay algo dentro de todo el mundo que nos empuja a buscarla, el "eros", según Platón. El filósofo debe despertarlo. Esas "grandes palabras" se refieren a esa sensación de pérdida cuando recordamos la ilusión con la que esperábamos la vida adulta en nuestra infancia, o la de preguntarnos "¿esto es todo?" en los raros momentos de silencio interno y externo en medio de nuestro acelerado modo de vida. Buscamos una vida más plena y en eso es, precisamente, en lo que puede contribuir la Práctica Filosófica: No en proporcionar satisfacción, ni en solucionar problemas (de timidez, con el jefe, etc.) sino en ayudar a vivir una vida plena. Para ello Lahav recorre dos fases:

- Una, dentro de la Filosofía, basada en el análisis de los límites inconscientes que imponemos a nuestra vida, en "entender la caverna y sus sombras".

- Y otra "más allá de la Filosofía", una fase ya no analítica, sino espiritual, en la que trabajamos en la transformación para ir más allá de los límites de la caverna, el "perímetro" en el que nos encerramos.

Esta segunda fase está basada en las ideas de que "estamos en el exilio" (coincidencia con lo dicho por Abellán anteriormente) y de que es posible esa transformación. El "camino del entendimiento" es el método para conseguirla que propone Lahav, escuchando la voz interior que nos evoquen los textos de una serie de filósofos, que denomina "internos", de épocas diferentes y aparentemente heterogéneos, pero que expresan esa voz que es el anhelo de vivir con plenitud: Marco Aurelio, Rousseau, Emerson, Bubber, Bergson, Fromm, Marcel, etc.

Para terminar nos emplazó a asistir a su taller del domingo, donde explicaría esta segunda fase con más detalle y con ejemplos prácticos, y sugirió que, mientras, pensásemos sobre esa voz interior. 
Sentido de la Filosofía o Filosofía del sentido, por PEDRO ORTEGA.

Emotiva fue esta charla en la que Ortega nos mostró que la Filosofía como terapia puede "curar el sinsentido", porque "cuando no hay pensamiento no hay sentido de la vida". Por eso, parafraseando a Aristóteles, afirmó que la Filosofía no sirve para nada, pero añadió que "para nada más que para aprender a vivir". Recordó la función terapéutica de la Filosofía ya en el mito de la caverna y para autores como Cicerón, Epicuro o el mismo Jung, que la consideró "la psiquiatría del futuro", ya que "la locura es la pérdida del sentido común", y la Filosofía trata con las "vidas mal planteadas", con el autoengaño que reacciona contra lo que cree que es la realidad. Para todo ello señaló cinco puntos:

1. El diálogo ha de ser incesante.

2. Es necesario aprender a pensar: expresar y sentir lo pensado.

3. Aprender a pensar es fuente de salud. La mala relación pensamientorealidad es la causa de la mayoría de las consultas al psicólogo.

4. Superar ese sufrimiento nos depara una alegría proporcional. La culpa, el mirar hacia atrás sólo es buena si es lo que nos permite pedir perdón, pero lo que ha de reinar es la responsabilidad, el mirar hacia adelante. Que llegue el reinado de la razón, y no de la culpa, como ya propuso Freud.

5. La Metafísica es necesaria para la Psicoterapia. Hay que ver que no son iguales la realidad sentida y la pensada, el error y la mentira, etc.

Uniendo pensamiento-sentimiento-realidad, que en verdad son inseparables, la Filosofía ayuda a poner en valor el sentido de la vida.

\section{Metodología y Ontología en la Práctica Filosófica, por TOMÁS ELLAKURÍA ${ }^{15}$.}

Hace doce años la obra de Antonio González ${ }^{16}$ marcó su propuesta metodológica, que se complementa con elementos de meditación y Psicología profunda. Lamentablemente, la falta de tiempo nos privó de poder conocerla en profundidad, ya que sólo pudo leer rápidamente las

\footnotetext{
${ }^{15}$ Filósofo asesor y auxiliar psiquiátrico, iniciado en la práctica del Zen.

${ }^{16}$ GONZÁLEZ, Antonio: Estructuras de la praxis, Trotta, Madrid, 1997.
} 
primeras consideraciones del texto que tenía preparado. Más tarde hemos podido tener acceso al texto íntegro, y hemos comprobado que en autores como Zubiri, Husserl y Blay, pero sobre todo el mencionado González, está la base de su metodología. El texto termina con una cuestión vinculada con el sentido de toda la charla, en sus palabras: “¿Existe la posibilidad de una práctica filosófica de raigambre científica, en cuanto a sus bases epistemológicas? Probablemente esto sí pueda ser una realidad.".

Práctica Filosófica y música, por MARIANO BETÉS DE TORO ${ }^{17}$.

En la sesión de mañana, Betés había hecho un breve avance de su conferencia, mientras comentaba las piezas musicales que sonaron, hablando de la función terapéutica, filosófica y social de la música.

Ya en la conferencia de la tarde, detalló cinco claves en musicoterapia:

1. Restablece la "armonía" espiritual. Punto estudiado desde Pitágoras.

2. Además de la función individual, la música tiene una función social, educativa, de doble filo.

3. No existe un mundo sin sonidos. Aunque continuamente focalizamos sólo en algunos.

4. Respondemos a la música, tanto consciente como inconscientemente.

5. Lo más importante: la música nos sirve para el autoconocimiento, por la catarsis y por la mezcla de recuerdos y sentimientos, positivos y negativos, que hace surgir, y que varían según el tipo de música, nuestra experiencia y el momento psicológico (mañana, noche...).

Al hacer conscientes estos sentimientos y recuerdos, la música nos permite analizar qué, por qué y cómo los sentimos. Para ese análisis un filósofo puede ser de gran ayuda, así como un experto en musicoterapia.

\footnotetext{
${ }^{17}$ Filósofo asesor, Doctor en Medicina, Licenciado en Psicología y en Historia del Arte, Catedrático de la Universidad de Alcalá. Magister y responsable de los postgrados en Musicoterapia de la Universidad de Alcalá.
} 
Filosofía Aplicada desde el abismamiento zambraniano, por JOSÉ BARRIENTOS ${ }^{18}$.

En el escaso tiempo disponible nos dio un rápido resumen de su actual visión en dos fases de la Filosofía Aplicada o Interventiva:

1. FASE LÓGICO-ARGUMENTAL. Es la metodología de Óscar Brenifier, Tim Le Bon, Elliot Cohen, Warren Shibles, entre otros. El paso de lo racional a lo vivido no se facilita, de las ideas a las creencias, sobre las que vivimos según Ortega. María Zambrano trabajó sobre ello y, sobre ese trabajo, Barrientos propone una segunda fase.

2. FASE EXPERIENCIAL. Para "saber" lo conocido, o descubierto, en la primera fase, "como quien tiene su primer hijo y dice que ya sabe lo que es ser padre", existe una vía estética y otra experiencial. Estética, por ejemplo, sería la descrita por Betés a través de la música, o mediante novelas como las nivolas de Unamuno, "Abel Martín", etc. La forma experiencial, sería haciendo vivir más conscientemente, por ejemplo, contó el caso en el que aconsejó al consultante que en lugar de resistirse a sus ataques de pánico nocturno, los describiese con detalle por escrito, con lo que disminuyeron y terminaron por desaparecerer. Otros ejemplos de este tipo serían una visita al cementerio, para experienciar la muerte, o los métodos expuestos por Betés en su paso por el programa de televisión "Terapia de pareja". Que estas vías lleven a buen puerto depende del consultante, en palabras de Zambrano, se le lleva hasta el "claro del bosque", pero sólo el propio consultante puede quedar iluminado.

Como ejemplo de estas diferentes maneras de conocer algo, usó el concepto "perseverancia", que puede ser conocido de forma racional, mediante la definición del diccionario, de forma estética, para lo que nos mostró un divertido vídeo musical que ilustraba perfectamente el término, o de forma experiencial, ilustrada con un emotivo fragmento del programa de Ophra Winfrey, en el que se plasmaba el mensaje "un obstáculo te demuestra cuánto quieres conseguir algo".

\footnotetext{
${ }^{18}$ Orientador Filosófico. Consejo Superior de Invetigaciones Científicas. Universidad de Sevilla
} 
Para terminar, Barrientos parafraseó a Giner de los Ríos para matizar que, si bien la Filosofía por sí misma da luz pero no calor, en el caso de Zambrano sí que es capaz de ambas cosas.

\section{Café filosófico, por Óliver Álvarez ${ }^{19}$. Sala de la Cacharrería.}

Unas cincuenta personas se congregaron para conocer la vertiente de los cafés filosóficos de la mejor forma posible, participando en uno de ellos. Aproximadamente la mitad de los presentes participaron activamente, de forma ordenada y con aportaciones concisas y bien expuestas. Se notó la abundancia de titulados en Filosofía entre el público, pero todas las citas que surgieron fueron suficientemente aclaradas para que todos los presentes siguieran sin dificultad el hilo de la conversación. Más de una docena de los habituales del café vallisoletano del bar Penicilino acudieron a la cita en Madrid para no perderse su sesión mensual, y colaboraron, además de con sus interesantes puntos de vista, con una muy buena demostración de la dinámica de los cafés coordinados por el autor de esta nota.

La sesión comenzó con una breve historia de los cafés filosóficos, desde el del profesor Marc Sautet, en París en 1992, y con una explicación de la mecánica que usaríamos en el nuestro en concreto, basada en una primera fase de ejemplos, y una segunda de coloquio con la definición del diccionario de la Real Academia como base, definición que no nos compromete a priori con ninguna opción filosófica, sino con la noción que la Academia detecta que es la que poseen la mayoría de los hablantes del castellano. A partir de ahí se trabaja para matizarla, en caso de que no englobe todos los ejemplos expuestos, o se utiliza como apoyo para avanzar en las diferentes cuestiones que suscite el término elegido como tema para cada sesión. En esta sesión en concreto, el tema fue "el ser humano", y se estudiaron cuestiones como la característica emocional, además de racional, del hombre; la justificación, o no, del antropocentrismo; y si lo que nos caracteriza más es la libertad, la moral, lo sagrado, el habla...

\footnotetext{
${ }^{19}$ Promotor de los cafés filosóficos del café-bar Penicilino, en Valladolid, y autor de esta nota.
} 
La actividad más lúdica y divulgativa de la Práctica Filosófica, ésta de los cafés filosóficos, implicó a los presentes, que demostraron que la Filosofía es accesible a todos los públicos, y que con cualquier edad y nivel académico se puede participar, aclarando y exponiendo nuestras opiniones y argumentos, y escuchando y apreciando los de los demás, consiguiendo llevarnos a casa cuestiones sobre las que seguir reflexionando, que de otra forma no habríamos advertido, y que pueden llevarnos a contemplar y vivir la vida de otra forma, puede que más plena, pero, seguro, más examinada.

\section{Domingo. La forma de entender: Cómo abrirnos a una dimensión de plenitud, taller impartido por Ran Lahav}

En la conferencia del sábado, Lahav habló de dos partes en su práctica, la primera dedicada a descubrir los patrones que definen el perímetro en el que se encierra el consultante, y la segunda, a la que dedicó este taller de mañana y tarde, orientada a cómo abrir ese perímetro para vivir en una "dimensión de plenitud", como dice el título. Parte del supuesto de que todos sentimos un anhelo de plenitud en nuestro corazón, Lahav propone terminar con nuestro exilio, volver a nuestra "casa real", mediante una transformación, que es posible si se emprende una búsqueda personal, que podemos orientar escuchando la "voz", el mensaje, que nos evocan algunos textos de los que Lahav denomina "filósofos internos" No se trata de analizarlos, ni de estar de acuerdo con ellos, "no es exactamente filosofía", sino que va "más allá". Los autores con los que trabajamos y los mensajes evocados fueron estos:

ROUSSEAU. "Soy más de lo que soy". Tras una breve contextualización biográfica, leímos un párrafo del autor y realizamos una relajación, tras la que dibujamos libremente lo que nos hubiese evocado el texto. Hicimos una ronda mostrando y explicando nuestros bocetos. LAHAV. "Lo que soy es más grande", tras otra lectura, recordamos experiencias personales análogas a las descritas en el texto. BERGSON. "Afuera hay algo diferente, no fragmentado". Aunque nuestra vida es como el flujo continuo de un río, vivimos como en las hojas de la superficie, en 
fragmentos aislados. Lahav nos guió en una meditación en la que visualizamos que buceábamos bajo ese río. Luego, describimos lo que habíamos encontrado allí con tres palabras y, en grupos de tres, buscamos cómo aplicar todo lo visto, en la vida. EMERSON. "Ese yo ampliado está más cerca de la Realidad y la Sabiduría", y el yo aprisionado no tiene ningún techo que le separe de esa "super-alma". MARCEL. "Plenitud". Se termina por abandonar la identificación con la propia voluntad, porque el ser en plenitud no calcula, se da como un regalo continuamente. Lahav estableció la metáfora del pianista que, tras años de práctica, simplemente se deja llevar cuando toca.

Además de estos ejercicios con textos, realizamos otros dos:

- Usamos la mano, para escribir por ejemplo, sin concentrarnos en ello, luego concentradamente y por último siendo conscientes de todo mientras lo hacemos. Luego lo repetimos en parejas, hablando sin más, primero, luego imaginándonos vistos desde fuera $\mathrm{y}$, finalmente, conscientes de todo lo presente, incluyendo ruidos de fondo, etc. Un ejercicio aplicable en la vida cotidiana para reenganchar con ese yo ampliado que añoramos.

- El último ejercicio comenzó en grupos en los que comentamos alguna anécdota en la que nos hayamos sorprendido actuando, con malestar, fuera de nuestros patrones. Luego supusimos cómo habría sido la situación viviéndola desde el yo ampliado, no restringido. Finalizó la tarde visualizando esa situación sin malestar, en una última meditación más larga y profunda que las anteriores, de la que salimos conmovidos y con la sensación de haber aprehendido algo difícilmente expresable racionalmente.

Un importante punto que Lahav aclaró en un momento de la tarde, fue el de su analogía con el mito de la caverna de Platón. Puede malinterpretarse que lo que está fuera es lo que está bien y lo que está dentro de nuestro perímetro está mal, pero no es eso lo que Lahav quiere mostrar. Más bien, aclaró, se refiere a que debemos ser conscientes de que no somos un pequeño yo encerrado en los límites del perímetro de nuestra caverna, como creemos, sino que nuestro yo vive en la total plenitud ilimitada, tanto dentro como fuera de los límites de los patrones que nos autoimponemos. Lo que añoramos es vivir de esa manera, plenamente y sin limitaciones. En la primera parte de la práctica de 
Lahav, esos límites ilusorios se hacen conscientes, y se comprueba que se puede vivir fuera de ellos, en la segunda, la estudiada en este taller, abriéndonos a una auténtica "dimensión de plenitud", nuestra "verdadera casa".

\section{Lunes. El arte de preguntar. La conciencia, reconciliarse con la finitud del ser, taller impartido por Óscar Brenifier.}

La mañana estuvo dedicada a un EJERCICIO DE INTERPRETACIÓN de pequeñas expresiones, orientado a vislumbrar qué visión del mundo tiene quien las usa. Para Brenifier, "la Práctica Filosófica es como un arte marcial, hay que saber qué pasa en la mente del otro con detalles casi imperceptibles". Ese análisis de las palabras, "coartadas" que pretenden ocultar cosas, puede resultar brutal, porque el "método socrático te muestra tu ignorancia, y eso molesta", aunque "la agresión depende de cómo perciba cada uno las preguntas". "Los otros son útiles para que te muestren lo que dices y cómo lo dices, eso es Asesoramiento Filosófico".

- "NO NECESARIAMENTE" fue la primera expresión que analizamos, llegando a la conclusión de que es una peculiar forma "tener razón" siempre, en la que no se contraponen opiniones en pie de igualdad, si no con la absoluta e impersonal necesidad, que sería la base de una visión determinista del mundo.

- "A VECES", aunque similar a la anterior, es una expresión que según nuestra conclusión, denota un vínculo no con la necesidad, sino con la posibilidad, con lo que se puede deducir que, en este caso, sí se da importancia a la voluntad en la cosmovisión de quien la usa a menudo.

- “iHOMBRE!” fue la última expresión sobre la que trabajamos brevemente, bajo el supuesto de que revela una visión dogmática, con proposiciones tan evidentes que ni siquiera merece la pena que se enuncien.

Estos, son indicios provisionales que pueden confirmarse o desmentirse más adelante, pero a los que, en todo caso, se debe estar atento en la consulta. Otros recursos fueron mencionados durante la mañana, como 
evitar conceptos vacíos, por ejemplo "malentendido", que implica que, aunque erróneamente, se ha entendido algo, que se debería explicitar. Un recurso más fue el de buscar un "común denominador" para comparar dos proposiciones, expresándolas mediante la misma categoría gramatical.

En la sesión de tarde, Brenifier varió su intención de efectuar y analizar una consulta $\mathrm{y}$, viendo que la inquietud del grupo estaba más orientada al trabajo en el aula, propuso un ejercicio en el que, de cualquier forma, expuso recursos valiosos para ambas facetas.

PREGUNTAR A LA PREGUNTA ${ }^{20}$. Comenzamos con "¿puedo ir a Valladolid en tres horas?". Primero se escriben preguntas para aclararla. Después se comprueba si las preguntas son coherentes o no con la primera. Finalmente, las que hayan sido aceptadas se reducen a un concepto, por ejemplo "¿en qué transporte?" sería reducible a "medio". La segunda pregunta que utilizamos fue "¿cómo saber si podemos tener confianza en los demás?”. Viendo las dificultades que encontrábamos aquí, Brenifier propuso el recurso de utilizar los conceptos resultantes del primer ejercicio. Sorprendentemente las preguntas surgieron con facilidad, demostrando la propuesta platónica de que existen conceptos con capacidad transversal, de "transposición". Además de este recurso, usamos otros, como imaginar dos respuestas distintas para verificar la funcionalidad de una pregunta, usar las partículas interrogativas para crear buenas preguntas, y usar, pero no abusar, de "¿por qué haces esa pregunta?” y "¿qué significa alguno de sus términos?” como preguntas iniciales. En resumen, en este ejercicio "los alumnos ven qué es un concepto y aprenden a pensar antes de contestar".

\section{Conclusión}

En estas jornadas, se mostraron algunas de las vertientes y metodologías más relevantes en la Práctica Filosófica actual, pudiéndose comprobar su variedad, a la vez que su coherencia.

\footnotetext{
${ }^{20}$ Descrito en BRENIFIER, Óscar: El diálogo en clase, Ediciones Idea, S. C. Tenerife, 2005, páginas 105-111.
} 


\section{Bibliografía}

BRENIFIER, Óscar: El diálogo en clase, Ediciones Idea, S. C. Tenerife, 2005.

BRENIFIER, Óscar: "Filosofar es dejar de vivir", disponible on-line en http://www.brenifier.com/espanol/filosofar_es_dejar_de_vivir.htm (último acceso 28 de noviembre de 2009).

BRENIFIER, Óscar: Ni sí ni no. Un libro para entender los grandes contrarios del pensamiento, Ediciones SM, Madrid, 2008.

ARNAIZ, Gabriel: "El giro práctico de la Filosofía", en Diálogo Filosófico, número 68, Madrid, 2007. Págs. 170-206.

WITTGENSTEIN, Ludwig: Los cuadernos azul y marrón, Tecnos, Madrid, 2009.

MARINOFF, Lou: Más Platón y menos Prozac, Ediciones B, Barcelona, 2000.

CENCILLO, Luis: Cómo Platón se vuelve terapeuta: Respuesta a Marinoff, Ediciones Fundación, Madrid, 2002.

BARRIENTOS RASTROJO, José: Vectores zambranianos para una teoría de la filosofía aplicada a la persona, Vicerrectorado de investigación, Universidad de Sevilla, 2009.

GONZÁLEZ, Antonio: Estructuras de la praxis, Trotta, Madrid, 1997. BERGSON, Henri: Time and Free Will, Muirhead Library of Philosophy, Londres, 1971.

MARCEL, Gabriel: Obras selectas de Gabriel Marcel. I: El misterio del ser. El dardo. La sed. La señal de la Cruz, Editorial Biblioteca Autores Cristianos, Madrid, 2004.

EMERSON, Ralph Waldo: Ensayos, Espasa-Calpe, Madrid, 2001.

LAHAV Ran: "What Lu told me" y "Plenitude is not from you", disponibles on-line en http://www.trans-sophia.net/115845/What-LuTold-Me (último acceso 10 de diciembre de 2009).

LAHAV, Ran y DA VENZA TILLMANNS, Maria: Essays on Philosophical Counselling, University Press of America, New York, 1995. 